\title{
Analysis in Cardiac Stability over Thirty Minute Periods
}

\author{
Masaki Hoshiyama ${ }^{1}$, Alan Murray ${ }^{2}$ \\ ${ }^{1}$ Department of Education, Meisei University, Tokyo, Japan, and \\ Dept. of Pediatrics, The University of Tokyo \& Affiliated Hospitals, Tokyo, Japan \\ ${ }^{2}$ School of Electrical and Electronic Engineering, and Faculty of Medical Sciences, \\ Newcastle University, Newcastle upon Tyne, UK
}

\begin{abstract}
Cardiac stability with little change in heart rate is important when studying the effect of cardiac interventions. The aim of this study was to analyse changes in heart rate during conditions expected to be stable. Subjects were studied during quiet relaxation to allow analysis of cardiac stability over thirty minute periods. Ten subjects with no known cardiac disease were enroled, and gave ethical consent. Their ages were $42 \pm 15$ years (mean \pm standard deviation). Subjects were given time for normal relaxation, but without any controlled conditions. After the study was explained, subjects were then asked to sit quietly on an office chair, while a single channel ECG was recorded to a computer for offline analysis. After a few weeks, subjects were instructed briefly in Zen meditation techniques, and rerecorded in similar conditions. There was a tendency for heart rate to fall and RR intervals to increase over the 30 min period, but overall this was not statistically significant, due primarily to individual subject variation resulting from respiratory effects, especially with the deeper regular breathing during meditation. There were small but significantly reduced heart rates with increased RR intervals during the meditation session, in comparison with relaxed sitting: mean $R R$ interval increasing from $0.78 \pm 0.10 s$ to $0.87 \pm 0.13 s(p=0.002)$, with the mean minimum RR (associated with highest heart rate) increasing from $0.70 \pm 0.10 \mathrm{~s}$ to $0.74 \pm 0.10 \mathrm{~s}$ $(p=0.008)$. With preceding uncontrolled relaxation, cardiac changes over a 30 minute period were small, and meditation generated an additional small overall reduction in heart rate, with increased $R R$ intervals.
\end{abstract}

\section{Introduction}

Heart rate analysis has been used in many clinical studies, including for diabetes [1], heart transplant [2,3], Alzheimer's disease and vascular dementia [4], and sleep [5], as well as for studies of multifractal characteristics [6], variation over 24 hours [7], during sleep [8,9] and Zen meditation [10].

For such studies, it is important to have information on stability of heart rate over extended recording periods.

We therefore in this study chose to analyse stability over 30 minute periods.

In addition, we also analysed the effect of meditation in comparison with relaxation.

\section{Methods}

ECGs were recorded over a 30-min period, and analysed for changes in heart rate for all subjects, and the effect of meditation analysed.

\subsection{Study subjects}

Ten subjects with no known cardiac disease were enroled, and gave ethical consent. Their ages were $42 \pm 15$ years (mean \pm standard deviation).

None of the subjects exhibited any ectopic beat during the recording periods.

\subsection{Study conditions}

Subjects were given time for normal relaxation, but without any controlled conditions. After the study was explained, subjects were then asked to sit on an office chair, while a single channel ECG was recorded to a computer for offline analysis. After a few weeks, subjects were instructed briefly in Zen meditation techniques including respiratory exercise for lower abdominal muscle respiration [10], and rerecorded in similar conditions.

Subjects were asked to remain seated and still, without talking for the 30 minute recording period.

\subsection{ECG recordings}

Single lead ECGs were obtained with three electrodes applied directly to the chest. The ECG amplifier gain was set at 1000 . The output of the amplifier was connected to 
an analogue-to-digital converter at a sample rate of $250 \mathrm{~Hz}$, and stored to a computer for off-line analysis.

\subsection{QRS detection}

ECG were filtered using time domain Savitzky-Golay filtering [11,12] and each ECG beat was detected by identifying the fast response associated with each QRS as well as by coarse grained local maxima procedures, followed by a cardiologist's confirmation.

\subsection{Heart rate analysis}

The inter beat RR intervals were determined and the instantaneous inter beat heart rate calculated.

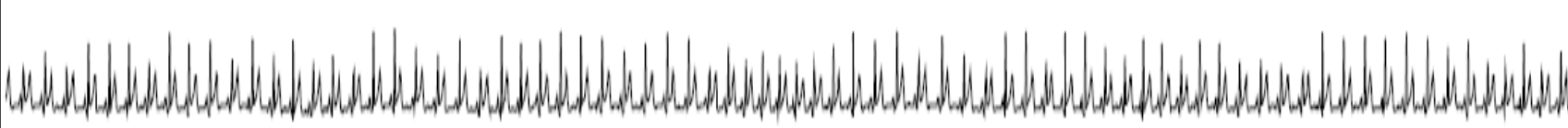

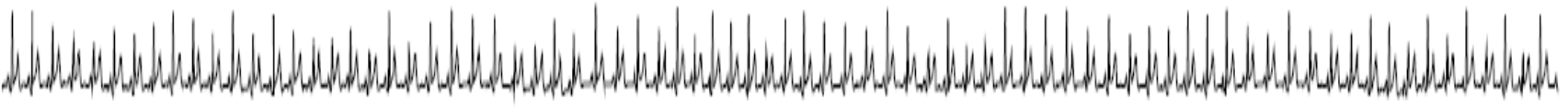 .

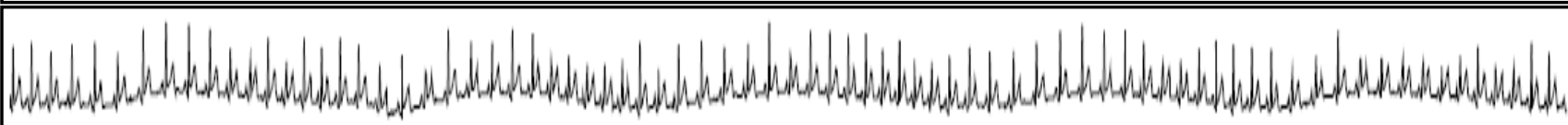 . (4)}

Figure 1. An ECG example from relaxation (above) and during meditation (below).

Three minutes from each 30-min recording is shown. It can be seen that regular respiratory changes are larger during meditation. 


\section{Results}

\subsection{Heart rate changes over $30 \mathrm{~min}$ period}

There was a tendency for heart rate to fall and RR intervals to increase over the $30 \mathrm{~min}$ period, but overall this was not statistically significant, due primarily to individual subject variation resulting from respiratory effects, especially with the deeper regular breathing during meditation (Figures 1 and 2).

\subsection{Comparison of heart rate between relaxation and meditation}

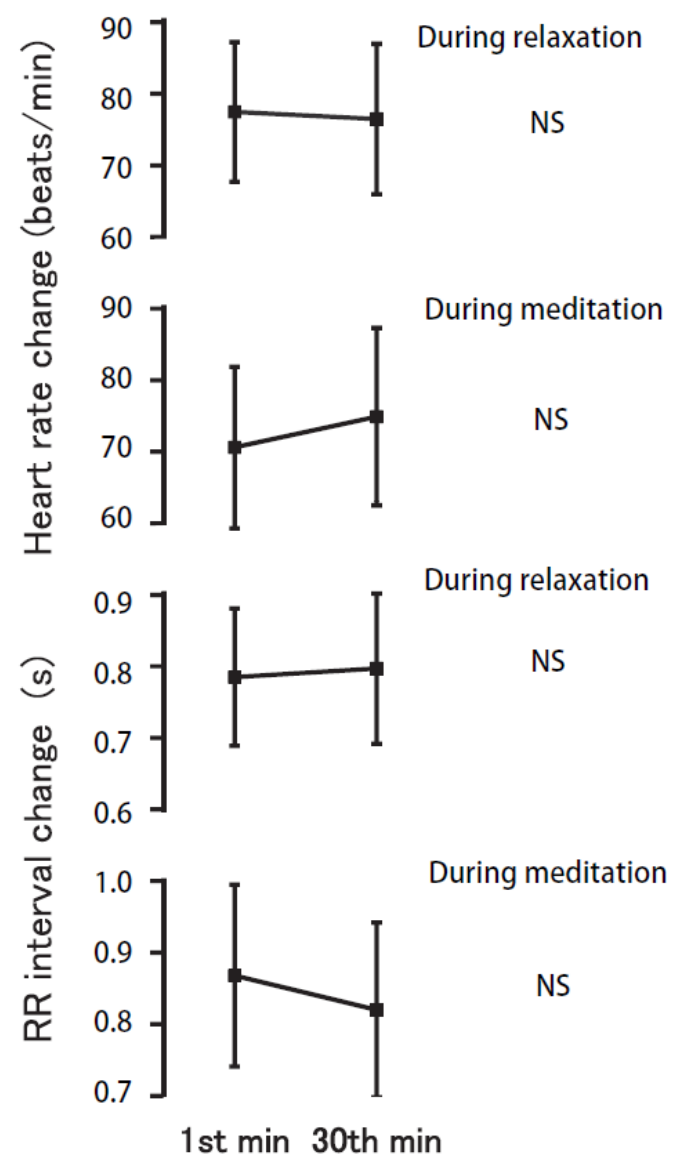

Figure 2. Change in instantaneous heart rate and RR over the 30-min periods during relaxation and during meditation.
There were small but significantly reduced heart rates with increased RR intervals during the meditation session, in comparison with relaxed sitting: mean RR interval increasing from $0.78 \pm 0.10 \mathrm{~s}$ to $0.87 \pm 0.13 \mathrm{~s}(\mathrm{p}=0.002)$, with the mean minimum RR (associated with the mean maximum instantaneous heart rate) increasing from $0.70 \pm$ $0.10 \mathrm{~s}$ to $0.74 \pm 0.10 \mathrm{~s}(\mathrm{p}=0.008$ ) (Figure 3).

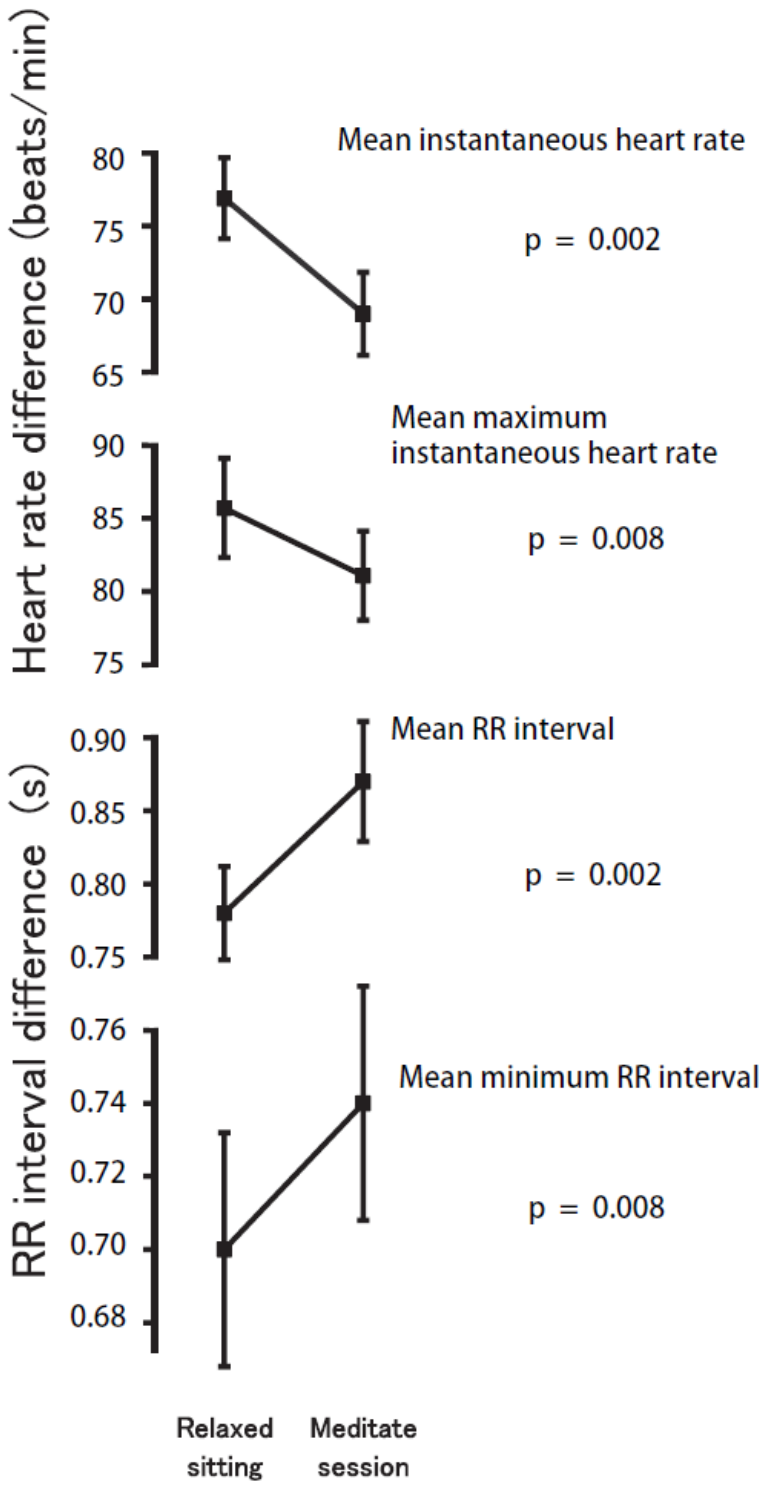

Figure 3. Paired difference for mean instantaneous heart rate and mean $\mathrm{RR}$, between relaxed and meditation periods. 


\section{Discussion and conclusion}

With the uncontrolled relaxation preceding the ECG recording periods, cardiac changes over a 30 minute period were small. All subjects had time to stabilise before the recording period, and in some case this period was over an hour. Therefore, as expected further changes were small.

When mean heart rate for the relaxed period was compared with the meditation period, it was shown that meditation generated a small overall reduction in heart rate, with increased RR intervals.

\section{Acknowledgement}

We acknowledge the support of the Great Britain Sasakawa Foundation.

\section{References}

[1] Lawrence GP, Home PD, Murray A. Repeatability of measurements and sources of variability in tests of cardiovascular autonomic function. British Heart Journal 1992;68:205-11.

[2] Lord SW, Clayton RH Mitchell L, Dark JK, Murray A, McComb JM. Sympathetic reinnervation and heart rate variability after cardiac transplantation. Heart 1997;77:5328.

[3] Lord SW, Senior RR, Das M, Whittam AM, Murray A, McComb JM. Low-frequency heart rate variability: reproducibility in cardiac transplant recipients and normal subjects. Clinical Science 2001;100:43-6.

[4] Allan LM, Kerr SRJ, Ballard CG, Allen J, Murray A, McLaren AT, Kenny RA. Autonomic function assessed by heart rate variability is normal in Alzheimer's disease and vascular dementia. Dementia and Geriatric Cognitive Disorders 2005;19:140-4.

[5] Drinnan MJ, Allen J, Langley P, Murray A. Detection of sleep apnoea from frequency analysis of heart rate variability. Computers in Cardiology 2000;27:259-62.
[6] Ramchurn SK, Murray A. Multifractal analysis of the day and night characteristics of heart rate variability. Computers in Cardiology 2002;29:421-4.

[7] Langley P, Allen J, Bowers EJ, Drinnan MJ, Haigh AJ, King ST, Olbrich T, Smith FE, Zheng D, Murray A. The ebb and flow of heart rate variability: simulation of 24 hour heart rate time series using time series data from naturally occurring phenomena. Computers in Cardiology 2005;32:973-6.

[8] Bunde A, Havlin S, Kantelhardt JW, Penzel T, Peter JH,Voigt K. Correlated and uncorrelated regions in heartrate fluctuations during sleep. Phys Rev Lett 2000;85:37369.

[9] Hoshiyama M, Hoshiyama A. Heart rate variability associated with rapid eye movements during sleep. Computers in Cardiology 2007;34:689-692.

[10] Hoshiyama M, Hoshiyama A. Heart rate variability associated with different modes of lower abdominal muscle tension during Zen meditation. Computing in Cardiology 2014; 41:773-776.

[11] Savitzky A, Golay MJE. Smoothing and differentiation of data by simplified least squares procedures. Analytical Chemistry $1964 ; 36: 1627-39$.

[12] Steiner J, Deltour J. Smoothing and differentiation of data by simplified least square procedure. Analytical Chemistry 1972; 44:1906-9.

Addresses for correspondence:

Professor Masaki Hoshiyama,

Department of Education, Meisei University, Tokyo, Japan M.Hoshiyama@gmail.com

Professor Alan Murray

School of Electrical and Electronic Engineering, and Faculty of Medical Sciences, Newcastle University, Newcastle upon Tyne, UK.

alan.murray@ncl.ac.uk 